\title{
Early onset seizures and Rett-like features associated with mutations in CDKL5
}

Julie C Evans* ${ }^{* 1,10}$, Hayley L Archer ${ }^{1,10}$, James P Colley ${ }^{1}$, Kirstine Ravn ${ }^{2}$, Jytte Bieber Nielsen ${ }^{3}$, Alison Kerr ${ }^{4}$, Elizabeth Williams ${ }^{1}$, John Christodoulou ${ }^{5}$, Jozef Gécz ${ }^{6}$, Philip E Jardine ${ }^{7}$, Michael J Wright ${ }^{8}$, Daniela T Pilz ${ }^{1}$, Lazarus Lazarou ${ }^{1}$, David N Cooper ${ }^{1}$, Julian R Sampson ${ }^{1}$, Rachel Butler ${ }^{1}$, Sharon D Whatley ${ }^{9}$ and Angus J Clarke ${ }^{1}$

\footnotetext{
${ }^{1}$ Department of Medical Genetics, Cardiff University, Heath Park, Cardiff, UK; ${ }^{2}$ Department of Clinical Genetics, University Hospital, Rigshospitalet, Copenhagen, Denmark; ${ }^{3}$ The John F Kennedy Institute, Glostrup, Denmark; ${ }^{4}$ Department of Psychological Medicine, University of Glasgow, Glasgow, UK; ${ }^{5}$ Western Sydney Genetics Program, The Children's Hospital at Westmead and Discipline of Paediatrics and Child Health, University of Sydney, Sydney, Australia; ${ }^{6}$ Department of Genetic Medicine, Women's and Children's Hospital and Department of Paediatrics, University of Adelaide, Adelaide, Australia; ${ }^{7}$ Department of Neurology, Bristol Royal Hospital for Sick Children, Bristol, UK; ${ }^{8}$ Institute of Human Genetics, International Centre for Life, Newcastle Upon Tyne, UK; ${ }^{9}$ Department of Medical Biochemistry, Cardiff University, Heath Park, Cardiff, UK
}

Mutations in the CDKL5 gene (also known as STK9) have recently been shown to cause early onset epilepsy and severe mental retardation (ISSX or West syndrome). Patients with CDKL5 mutations sometimes also show features similar to those seen in Rett Syndrome (RTT). We have screened the CDKL5 gene in 94 patients with RTT or a RTT-like phenotype who had tested negative for MECP2 mutations (13 classical RTT female subjects, 25 atypical RTT female subjects, 40 RTT-like female and 16 RTT-like male subjects; 33 of the patients had early onset seizures). Novel pathogenic CDKL5 mutations were identified in three girls, two of whom had initially been diagnosed with the early onset seizure variant of RTT and the other with early onset seizures and some features of RTT. In addition, the 33 patients with early seizures were screened for the most common mutations in the $A R X$ gene but none were found. Combining our three new cases with the previously published cases, 13/14 patients with CDKL5 mutations presented with seizures before the age of 3 months.

European Journal of Human Genetics (2005) 13, 1113-1120. doi:10.1038/sj.ejhg.5201451;

published online 13 July 2005

Keywords: CDKL5; STK9; ARX; ISSX; West syndrome; Rett; epilepsy

\section{Introduction}

Several recent papers have described mutations in the X-linked gene cyclin-dependent kinase-like 5 (CDKL5,

${ }^{*}$ Correspondence: Dr JC Evans, Department of Medical Genetics, Cardiff University, Heath Park, Cardiff, CF14 4XN, UK.

Tel: + 44292074 4673; Fax: + 44292074 7603;

E-mail: evansjc6@cardiff.ac.uk

${ }^{10}$ These authors contributed equally to this work

Received 8 March 2005; revised 21 April 2005; accepted 17 May 2005; published online 13 July 2005
OMIM\#300203), previously known as serine/threonine kinase 9 , in patients with seizures presenting in the first few months of life and severe mental retardation. ${ }^{1-4}$ The combination of infantile spasms, hypsarrhythmia and severe mental retardation is known as West syndrome or, when X-linked, ISSX. (OMIM\#308350) The first CDKL5 mutations were found in two girls with ISSX and X;autosome translocations. In both cases, the breakpoints disrupted the CDKL5 gene at Xp22.3. ${ }^{1}$ Subsequently reported CDKL5 mutations have been shown to produce 
a similar phenotype with an X-linked dominant inheritance pattern. ${ }^{2-4}$ Mutations in the Aristaless (ARX) gene represent another cause of early onset epilepsy with mental retardation. ${ }^{5}$ Approximately $80 \%$ of $A R X$ mutations occur in exon 2 and there is a common exon 2 mutation, $428-451$ dup (24 bp), that accounts for $30 \%$ of all known mutations (JG personal communication).

Some of the patients reported with CDKL5 mutations had initially been diagnosed with atypical Rett Syndrome (RTT, OMIM\#312750). RTT is a neurodevelopmental disorder that predominantly affects girls and is the most common genetic cause of profound intellectual disability in females. Typically there is a period of near normal development followed by regression with loss of social, motor and communication skills. Other features of RTT include hand stereotypies, microcephaly, autonomic disturbance, scoliosis, feeding difficulties and epilepsy. Up to 95\% of classical RTT and $40-50 \%$ of atypical RTT cases are caused by mutations in the X-linked gene methyl-CpG binding protein 2 (MECP2, OMIM\#300005). ${ }^{6,7}$

Atypical RTT can be divided into several different subgroups. One of these, known as the Hanefeld variant, describes female patients with atypical RTT and infantile spasms. ${ }^{8}$ This variant was extended to include those with early onset of epileptic seizures. ${ }^{9}$ Seizures of early onset, or of onset before regression, are an unusual feature in RTT. As far as we are aware, no MECP2 mutations have been reported in patients with infantile spasms and features consistent with RTT. This suggests that most cases of RTT with early onset seizures are caused by a different mechanism.

The aim of this study was to determine how frequent CDKL5 mutations are in the different subtypes of RTT and in which patients CDKL5 testing should be considered. We screened the CDKL5 gene for mutations in a total of 94 patients with RTT or RTT-like features who had previously tested negative for MECP2 mutations. In addition, we tested the 33 patients who had early onset seizures for mutations in exon 2 of $A R X$. We describe three patients with novel pathogenic CDKL5 mutations and review the clinical features of the patients published to date.

\section{Methods \\ Samples}

Blood DNA samples were obtained from 94 patients with suspected RTT. Most were recruited from patients who had been referred to the Medical Genetics Service for Wales for MECP2 testing and from patients on the British Isles Rett Syndrome Survey who were reported to have early seizures. Six samples were obtained from Denmark. Clinical characteristics of the patients are shown in Table 1. All samples have previously proven negative for MECP2 mutations by sequencing exons 1-4 and by using Multiplex Ligationdependent Probe Amplification (MRC Holland) or quanti-
Table 1 Clinical characteristics of the patients included in this study

\begin{tabular}{ll}
\hline Rett type & Number \\
\hline Classical Rett females & 13 \\
Atypical Rett females & 25 (15 with early onset seizures) \\
Rett-like females & 40 (15 with early onset seizures) \\
Rett-like males & 16 (3 with early onset seizures) \\
Total & 94 (33 with early onset seizures) \\
\hline
\end{tabular}

Patients were classified according to the criteria in Hagberg et al. ${ }^{12}$ Patients described as 'Rett-like' had some features of RTT but did not fulfil the criteria for diagnosis or were not old enough to be classified. Early seizures were defined as seizure onset in the first 12 months of life.

tative fluorescent PCR to detect large deletions. ${ }^{10}$ Ethical approval was obtained for this study; MREC(Wales) Ref $02 / 9 / 33$.

\section{CDKL5 mutation screening}

Exons 1-21 of the CDKL5 gene were amplified by PCR. Primer sequences as described by Kalscheuer et al ${ }^{1}$ with a few exceptions: primers were redesigned if they were positioned less than $50 \mathrm{bp}$ from the exon (primer sequences available in Supplementary Table online). PCRs were optimised using Optimase Taq DNA polymerase and buffers (Transgenomic) according to the manufacturer's instructions. Mutation screening was performed by denaturing high-performance liquid chromatography (DHPLC) analysis (Transgenomic WAVE machine). Melting profiles and DHPLC run conditions were determined using Navigator software 1.5.3 (Transgenomic, details available in Supplementary Table online). Any shifts were checked by sequencing the PCR products using the PCR primers. Exons 1,4 and 16 were screened by DNA sequencing because of the higher rate of polymorphism in these exons.

\section{$A R X$ mutation screening}

Exon 2 was amplified in two sections using the following PCR primers: $A R X 2.1 \mathrm{~F} \quad 5^{\prime}$-cagcagcctggctgggactc- $3^{\prime}$, $A R X 2.1 \mathrm{R} \quad 5^{\prime}$-gtgccggtgccaccaccogcag-3', ARX2.2F $5^{\prime}$-cacg cacccggaggagcgcctc- $3^{\prime}, \quad A R X 2.2 \mathrm{R} \quad 5^{\prime}$-tgcgctctctgccgctgcgac $-3^{\prime}$. PCR was carried out in a $\left[\mathrm{NH}_{4}\right]_{2} \mathrm{SO}_{4}$-based buffer with $3.7 \mathrm{mM} \mathrm{MgCl}_{2}, 750 \mu \mathrm{M}$ dNTPs, $0.5 \mathrm{M}$ betaine, 5\% DMSO and platinum Taq DNA polymerase (Invitrogen). The annealing temperature was $65^{\circ} \mathrm{C}$. Sequencing was performed with the same primers using a BigDye v1.1 sequencing kit (Applied Biosystems) according to the manufacturer's instructions except for the addition of $1 \mathrm{M}$ betaine to the sequencing reactions.

\section{cDNA analysis of splice site mutations}

Total RNA was extracted from lymphocytes using the QIAamp RNA blood mini kit (Qiagen). RNA was converted to cDNA using a superscript first-strand synthesis system for RT-PCR (Invitrogen). PCR primers were designed to 
span several exons flanking the splice site variant and used to amplify the cDNA (primer sequences available in Supplementary Table online). RT-PCR products were run on a $2 \%$ agarose gel until the two bands separated. The RTPCR bands were excised from the agarose, purified using a QIAquick Gel Extraction Kit (Qiagen) and sequenced using the PCR primers.

\section{$\mathrm{X}$-inactivation}

$\mathrm{X}$-inactivation ratios were determined using standard methods. ${ }^{11}$ Briefly, separate aliquots of DNA were digested using the methylation-sensitive restriction enzymes HpaII and $\mathrm{McrBC}$ (New England Biolabs). The polymorphic repeat at the androgen receptor locus was then amplified using fluorescent PCR primers and the allele peak areas analysed using an ABI 3100 automated sequencer and Genotyper software (Applied Biosystems).

\section{Results}

\section{CDKL5 and $A R X$ mutation screening}

A total of 15 novel CDKL5 variants were identified (Table 2). Of particular interest were two splice site mutations (IVS7$2 \mathrm{~A}>\mathrm{G}$ and IVS16 $+1 \mathrm{G}>\mathrm{C}$ ) and three missense mutations (I72N, H467R and R444C). None of the 33 patients with early onset seizures had mutations in exon 2 of $A R X$.
Splice site mutation IVS7-2A $>$ G

This mutation disrupts the AG dinucleotide of the splice acceptor site of exon 8 and was found in patient 1 (Figure 1). Neither of the patient's parents had the mutation. Amplification of exons 6-10 from this patient's cDNA produced two different sized RT-PCR products; one was approximately $100 \mathrm{bp}$ smaller than predicted. This would be consistent with the loss of exon 8 (91 bp). Sequencing of the smaller RT-PCR product confirmed that exon 8 was skipped which would result in a frameshift. Translation of this abnormal RNA would be predicted to lead to the addition of 42 abnormal amino acids after amino-acid 155 and then premature truncation of the protein (G155fsX197). The X-inactivation assay was not informative in this patient but the relative signal strengths obtained for the two RT-PCR products on an agarose gel (Figure 1b) and by sequencing (not shown) were similar, suggesting $\mathrm{X}$-inactivation was random.

Splice site mutation IVS16 $+1 \mathrm{G}>\mathrm{C}$

This mutation disrupts the GT dinucleotide of the splice donor site of exon 16 and was found in patient 2 (Figure 2). The mutation was not present in either parent. Amplification of exons 13-18 from this patient's cDNA produced two different sized RT-PCR products; one was approximately $100 \mathrm{bp}$ smaller than predicted. This would be

Table 2 CDKL5 sequence variants identified to date

\begin{tabular}{|c|c|c|c|}
\hline Type of variant & Sequence change & Comments & Reference \\
\hline Splice site & $\begin{array}{l}\text { IVS7-2A }>G \\
\text { IVS13-1G }>A \\
\text { IVS16+1G }>C\end{array}$ & $\begin{array}{l}\text { Pathogenic, results in exon } 8 \text { skipping (patient } 1 \text { ) } \\
\text { Pathogenic, activates an alternative splice acceptor site } \\
\text { Pathogenic, results in exon } 16 \text { skipping (patient 2) }\end{array}$ & $\begin{array}{l}\text { This study } \\
\text { This study }\end{array}$ \\
\hline Deletion/insertion & $\begin{array}{l}\text { c. } 163 \_166 \text { delGAAA } \\
\text { c. } 183 \text { delT } \\
\text { c. } 2635 \_2636 \text { delCT }\end{array}$ & $\begin{array}{l}\text { Pathogenic } \\
\text { Pathogenic } \\
\text { Pathogenic }\end{array}$ & $\begin{array}{l}4 \\
2 \\
4\end{array}$ \\
\hline Missense & $\begin{array}{l}\text { c. } 215 \mathrm{~T}>\mathrm{A}(\mathrm{I} 72 \mathrm{~N}) \\
\text { C. } 455 \mathrm{G}>\mathrm{T}(\mathrm{C} 152 \mathrm{~F}) \\
\text { c. } 525 \mathrm{~A}>\mathrm{T}(\mathrm{R} 175 \mathrm{~S}) \\
\text { c. } 1330 \mathrm{C}>\mathrm{T}(\mathrm{R} 444 \mathrm{C}) \\
\text { C. } 1399 \mathrm{~A}>\mathrm{G}(\mathrm{H} 467 \mathrm{R}) \\
\text { c. } 2372 \mathrm{~A}>\mathrm{C}(\mathrm{Q} 791 \mathrm{P})\end{array}$ & $\begin{array}{l}\text { Pathogenic, conserved amino acid (patient 3) } \\
\text { Pathogenic } \\
\text { Pathogenic } \\
\text { Not pathogenic: present in unaffected father } \\
\text { Not pathogenic: present in unaffected mother } \\
\text { Known SNP }\end{array}$ & $\begin{array}{l}\text { This study } \\
3 \\
3 \\
\text { This study } \\
\text { This study }\end{array}$ \\
\hline Exon 1 and promoter ${ }^{a}$ & $\begin{array}{l}\text { c. }-440 \mathrm{G}>\mathrm{T} \text { (promoter) } \\
\text { c. }-391 \mathrm{G}>\mathrm{T} \text { (promoter) } \\
\text { c. }-265 \mathrm{C}>\mathrm{G} \text { (exon 1) } \\
\text { c. }-189 \mathrm{C}>\mathrm{T}(\text { exon } 1)\end{array}$ & $\begin{array}{l}\text { Present in } 1 \text { male patient, not seen in } 69 \text { controls } \\
\text { SNP: present in } 2 / 69 \text { male controls } \\
\text { SNP: present in } 2 / 69 \text { male controls } \\
\text { Present in } 1 \text { female patient, not seen in } 69 \text { controls }\end{array}$ & $\begin{array}{l}\text { This study } \\
\text { This study } \\
\text { This study } \\
\text { This study }\end{array}$ \\
\hline Intronic & $\begin{array}{l}\text { IVS4+4_5dupAT or delAT } \\
\text { IVS5-43G }>\text { A } \\
\text { IVS7-37_40delCTTT } \\
\text { IVS7+22T }>C \\
\text { IVS14+48C }>\text { T } \\
\text { IVS16+118T }>\text { A } \\
\text { IVS+17A }>\text { G }\end{array}$ & $\begin{array}{l}\text { Polymorphic [AT]n repeat, seen in } 3 / 37 \text { male controls } \\
\text { Present in } 1 \text { female patient } \\
\text { Present in } 1 \text { female patient } \\
\text { Present in } 1 \text { female patient } \\
\text { Present in } 1 \text { female patient } \\
\text { Common SNP (minor allele freq } 33 \% \text { ) } \\
\text { Rare haplotype with c.3003C }>\text { T and c.3084G }>\text { A }\end{array}$ & $\begin{array}{l}\text { This study } \\
\text { This study } \\
\text { This study } \\
\text { This study } \\
\text { This study } \\
\text { This study }\end{array}$ \\
\hline
\end{tabular}

${ }^{a}$ Exon 1 and promoter variants are numbered relative to the start codon in exon 2 . 
a
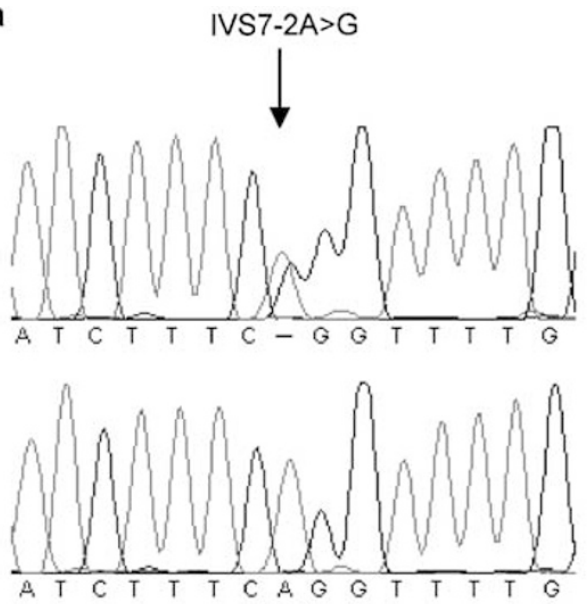

b $\quad 100 \mathrm{bp} \quad \mathrm{NM} \quad \mathrm{NN}$

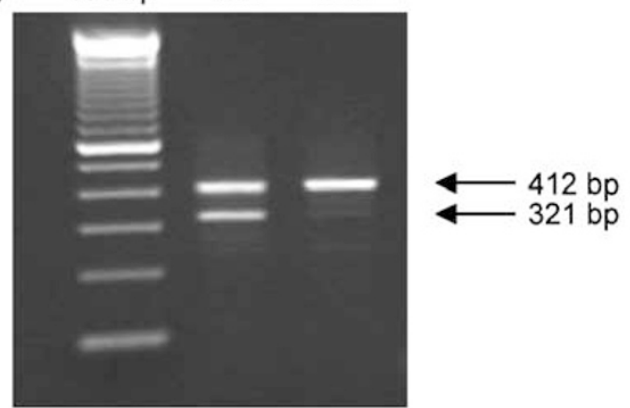

C
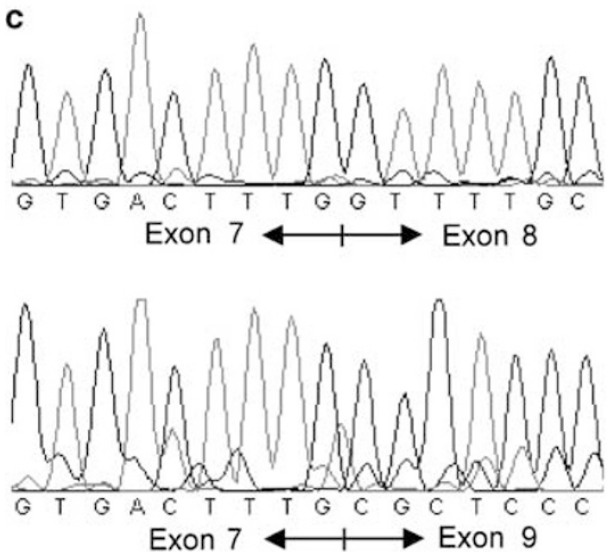

Figure 1 (a) Sequence trace showing the IVS7-2A $>G$ mutation in patient 1 (top) compared to a normal control (bottom). (b) RT-PCR products for patient $1(\mathrm{NM})$ and a normal control (NN) showing the presence of an abnormally sized cDNA in patient 1 . (c) Sequence trace of cDNA from patient 1 (bottom) and a normal control (top) showing skipping of exon 8. (d) (Online supplementary Information) Photograph of patient 1 aged 7 years.

consistent with the loss of exon 16 (100 bp). Sequencing of the smaller RT-PCR products confirmed that exon 16 was skipped which would result in a frameshift. Translation of this abnormal RNA would lead to the addition of nine abnormal amino acids after amino-acid 760 and then premature truncation of the protein (K760fsX769). a
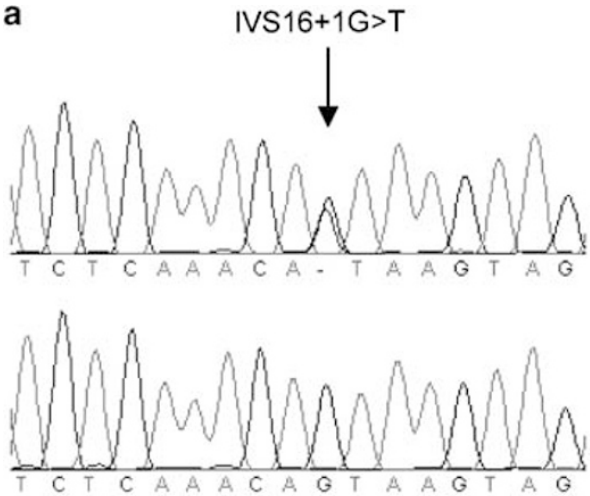

b 100 bp NM NN

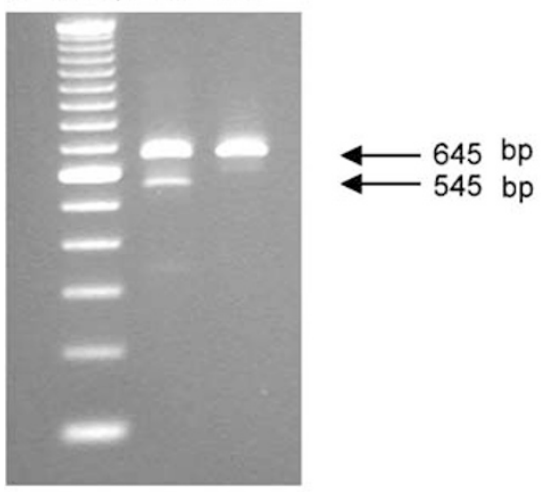

C
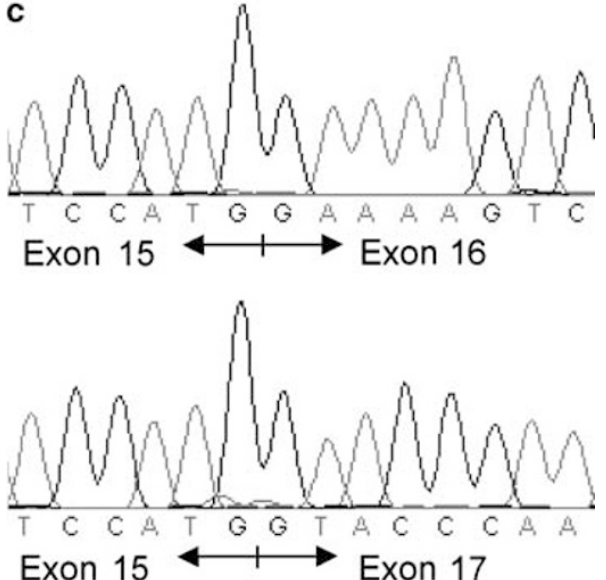

Figure 2 (a) Sequence trace showing the IVS16 + 1G > T mutation in patient 2 (top) compared to a normal control (bottom). (b) RT-PCR products for patient $2(\mathrm{NM})$ and a normal control $(\mathrm{NN})$ showing the presence of an abnormally sized CDNA in patient 2. (c) Sequence trace of cDNA from patient 2 (bottom) and a normal control (top) showing skipping of exon 16. (d) (Online Supplementary Information) Photograph of patient 2 aged 5 years.

$\mathrm{X}$-inactivation was random in this patient (52\%) but the mutant RT-PCR product was weaker on agarose gel (Figure 2b) and the signal strength on sequencing was approximately half that of the normal mRNA. This suggests that the mutant mRNA in this case may be more unstable than the normal mRNA. 


\section{Missense mutation (I72N)}

Patient 3 was heterozygous for a missense mutation in exon 5 (c.215T $>$ A, p.I72N, Figure 3). We screened both her parents and 184 control chromosomes and did not find the mutation (data not shown). This mutation occurs in the highly conserved serine/threonine kinase domain at the $\mathrm{N}$ terminus of the CDKL5 gene. The two other pathogenic CDKL5 missense mutations that have been reported were also located in this domain. ${ }^{3}$ The isoleucine at position 72 is strictly conserved in mouse, chicken and puffer fish (Figure 3b). Taken together, these observations strongly suggest that $\mathrm{I} 72 \mathrm{~N}$ is pathogenic. Patient 3 had moderately skewed X-inactivation (74\%).

\section{Nonpathogenic missense mutations}

DNA samples were obtained from the parents of the other two female subjects with missense mutations. The mother of the patient with H467R also had the variant. Xinactivation was not significantly skewed in leucocytes from either of them $(58 \%$ in the girl and $56 \%$ in her mother) so H467R is unlikely to be pathogenic. The father of the patient with $\mathrm{R} 444 \mathrm{C}$ was hemizygous for the variant implying that this change is not pathogenic.

\section{Noncoding variants}

A number of variants were found in the introns, the noncoding exon 1 and the promoter region of the CDKL5

a

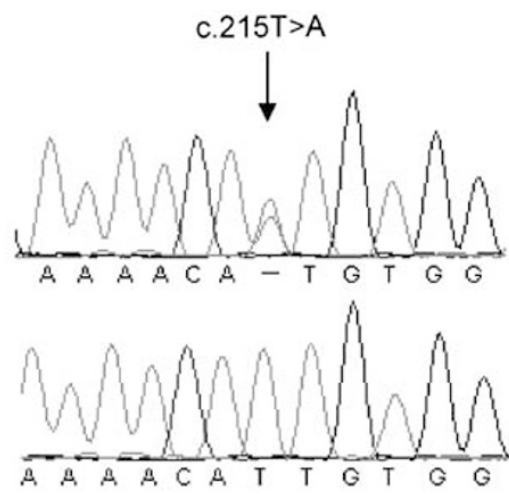

b

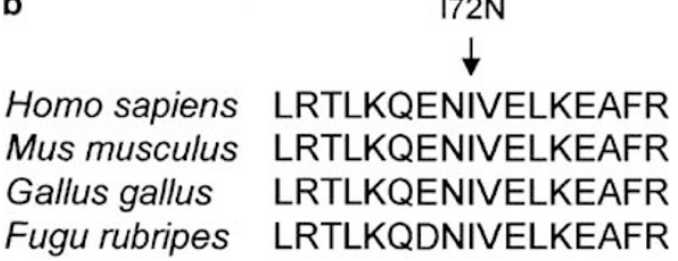

Figure 3 (a) Sequence trace showing the $172 \mathrm{~N}$ mutation in patient 3 (top) compared to a normal control (bottom). (b) Multiple alignment of $C D K L 5$ sequences showing strong sequence conservation around the $172 \mathrm{~N}$ mutation (GenBank accession numbers NM_003159, BAC34965, XP_42557 and AAD28798). (c) (Online Supplementary Information) Photograph of patient 3 aged 11 years. gene of other patients (Table 2). Some of these were also seen in unaffected controls and are therefore likely to be rare polymorphisms. None of the six intronic variants altered the splice site consensus sequences or created new splice sites according to the prediction software used (Berkeley Drosophila Genome Project website http:// www.fruitfly.org/seq_tools/splice.html and Splice Site Finder website http://www.genet.sickkids.on.ca/ ali/ splicesitefinder.html). Two of the four promoter/exon 1 variants were not seen in controls and, because family samples were not available, their significance is unclear. They do not disrupt any known cis-acting promoter sequences predicted by Proscan (http://thr.cit.nih.gov/ molbio/proscan). Sequence similarity between the human and mouse genomic sequence in this region is $85 \%$ for exon 1 and $75 \%$ for the first $480 \mathrm{bp}$ of the promoter.

\section{Clinical description of patient 1 (IVS7-2A $>$ G)}

Patient 1 was born following a normal pregnancy and delivery at 37 weeks, with a birth weight of $4.7 \mathrm{~kg}$ (98th centile) and head circumference of $34.0 \mathrm{~cm}$ (75th-91st centile). She had breast milk jaundice requiring one day of phototherapy on two separate occasions. She was a placid, sleepy baby and did not always wake for feeds. Her hands were always closed as an infant. At 3 weeks she had several episodes of trembling all over associated with unusual facial grimacing. She subsequently developed generalised tonic-clonic, absence and tonic seizures. She had a severe mixed seizure disorder that was refractory to medication, such that antiepileptic medications were withdrawn. Her EEG showed nonconvulsive status, but never showed hypsarrhythmia. Her MRI brain scan was normal. Her head circumference fell below the 3rd centile in her first year. She had severe gastro-oesophageal reflux and was predominantly fed by a gastrostomy tube. Her developmental progress was very limited. At 7.5 years, she had poor head control, rolled from back to side and only had fleeting eye contact. She had midline hand stereotypies, hand wringing and mouthing. She had variable tone, with increased tone on her left side. She had a mild lumbar scoliosis that was first noted at 4 years. She also had cold urticaria and small cold feet. Her weight and head circumference were below the 0.4 th centile and height on the 0.4 th-2nd centile. Additional features included large simple ears, wide spaced teeth with cusps on her incisors and delayed dentition, a divergent squint of her left eye, bilateral fifth finger clinodactyly and hyperconvex nails in both antero-posterior and lateral dimension. Whilst she fulfilled the Hagberg criteria for atypical RTT, ${ }^{12}$ clinically her phenotype was more consistent with a diagnosis of severe epileptic encephalopathy.

\section{Clinical description of patient 2 (IVS16 $+1 \mathrm{G}>\mathrm{C}$ )}

Patient 2 was born at term following a normal pregnancy, with a birth weight of $3.26 \mathrm{~kg}(9-25 \mathrm{th}$ centile) and head 
circumference of $33 \mathrm{~cm}$ (2nd centile). She was a placid, sleepy baby. She smiled at 6 weeks. She had recurrent episodes of facial grimacing at 5 weeks. At 8 weeks she had a prolonged tonic seizure whilst feeding. She subsequently developed salaam attacks. Her EEG initially showed hypsarrhythmia, but subsequently showed nonconvulsive status. Neuroimaging was normal. She had a severe, refractory, mixed seizure disorder, which consisted of grand-mal, absence, myoclonic seizures and eye rolling episodes. Her seizures were very resistant to treatment, but showed some improvement in frequency and duration in response to an implanted vagus nerve stimulator. Her medication included levetiracetam $70.4 \mathrm{mg} / \mathrm{kg} /$ day and clobazam $1 \mathrm{mg} / \mathrm{kg} /$ day. Her developmental progress was very poor following the onset of seizures. At 5.5 years, she could roll and lift her head against gravity. She had no purposeful hand use and used to wring her hands, but now rubs her head with one hand. She had marked hypotonia and a mild scoliosis, which was noted at 18 months of age. She only smiled out of context but could transiently fix and follow. She never developed speech but vocalised with a prolonged monotone. Her weight was on the $0.4-2$ nd centile and her height and head circumference were below the 0.4 th centile. She had small, cold feet. She had an unusually placed second toe, which was flexed and dorsally displaced. She also had wide-spaced teeth. At 5.5 years her diagnosis was consistent with ISSX.

\section{Clinical description of patient 3 (I72N)}

Patient 3 was the third child born to nonconsanguineous parents. She was born at term with a birth weight of $3.5 \mathrm{~kg}$ (50th centile) and head circumference of $35.5 \mathrm{~cm}$ (75th centile). There were no problems during the neonatal period, but she was very placid and sleepy. Seizures were noted at the age of 4 weeks but these were not infantile spasms. She smiled at the expected age and there were no concerns about her social interaction in the first 6 months, however, subsequently autistic features became apparent. Her babbling was limited and she never spoke words. She learnt to roll and to sit unsupported at 2 years and stand with support at 4 years. Midline hand stereotypies, hand wringing, clapping and mouthing, appeared at 3 years. She developed a mixed seizure disorder with generalised tonic-clonic and myoclonic jerks, refractory to treatment. Antiepileptic medication was stopped at 6 years of age because of limited effectiveness. Following this her motor development improved and she subsequently learnt to walk with support. At 10 years a mild scoliosis was noted. When assessed at 11 years of age, she had a mixed seizure disorder. She had irregular respiration (not hyperventilation or apnoea) with abdominal bloating. She also had screaming episodes and bruxism. She was particularly sensitive to loud noises and often blocked her ears with her fingers. No dysmorphic features were noted. Her height was on the 0.4 th-2nd centile, and weight and head circumference was below the 0.4 th centile. She had two MRI brain scans, the first at 8 months of age was normal, but a repeat scan at 3.5 years of age showed a small lesion in the right posterior hippocampal region. Clinically, she fulfilled the criteria for atypical RTT (early seizure variant).

\section{Discussion}

We have identified three patients with novel pathogenic mutations in the CDKL5 gene. This brings the total number of published mutations to 12 and the number of reported cases to $16 .^{1-4,13}$ All three patients in this study had initially been referred for MECP2 mutation testing although in retrospect patients 1 and 2 did not have RTT. Some CDKL5 patients have been reported to have atypical RTT but none presented with classic RTT. However, there are some similarities between the most severe CDKL5 phenotype and the male encephalopathic presentation of RTT. ${ }^{14}$ Two of our patients presented with a very severe phenotype, one of whom had ISSX. In common with other patients with a severe presentation they had limited, if any, eye contact which contrasts with the intense eye gaze of those with RTT. ${ }^{1-3}$ Poor eye contact was also apparent in some with a milder phenotype (patient 3 in this study, Weaving et $a l^{2}$ Tao et $a l^{3}$ ). Our patients did not have a classic regression period, and in those few where regression has been reported, this has often been episodic and in association with increased seizure activity. The timing of onset of seizures, presence of infantile spasms, lack of intense eye gaze and, if present, atypical regression may help to clinically distinguish patients with CDKL5 mutations on clinical grounds from those with MECP2 mutations.

Some of the common features of patients with CDKL5 mutations are shown in Table 3. Of 11 patients in the literature and three from this study with detailed clinical information, the most consistent clinical feature was early onset of seizures: 13/14 patients had seizures and in all of these the onset was at or before 3 months of age. Eight patients had infantile spasms, but two of these did not develop a subsequent epileptic seizure disorder. Of those who did, all had a severe intractable mixed seizure disorder, with the most frequently reported seizure type being generalised tonic-clonic seizures and myoclonic jerks. Two patients benefited from an implanted vagus-nerve stimulator (Tao et $\mathrm{al}^{3}$ and this study), but otherwise epilepsy was largely resistant to treatment with antiepileptic medication. Epileptic encephalopathy or epilepsy in the first 6 months of life are extremely unusual in female patients with clinical features of RTT and a MECP2 mutation. ${ }^{15}$ Infantile spasms have not been reported in association with a MECP2 mutation. 
Table 3 Summary of clinical features reported in patients with CDKL5 mutations

\begin{tabular}{ll}
\hline Diagnosis & $8 / 14$ atypical RTT (early seizure variant) \\
& $1 / 14$ severe infantile epileptic encephalopathy \\
& $4 / 14$ ISSX \\
& $1 / 14$ autism \\
Male:female ratio & $1: 13$ \\
Familial & $2 / 11$ families (2 pairs of identical twins) \\
Age range & $3-41$ years (mean 16 years) \\
Mortality & $3 / 13$ died (all before 18 years, and all ISSX) \\
X-inactivation status & Six skewed $(2,100 \% ; 6$, random; 1 , male; 1, not known) \\
Seizures ever & $13 / 14$ \\
Seizures at presentation & $13 / 14$ \\
Age at first seizure & Neonatal -3 months \\
Infantile spasms & $8 / 14$ \\
Ongoing epileptic seizures & $11 / 14$ \\
Subsequent seizure type & $7 / 14$ generalised tonic-clonic and myoclonic seizures \\
Poor seizure control & $11 / 14$ \\
Improvement in seizures & Two older patients improved with age \\
Hypsarrhythmia & Two benefited from a vagus-nerve stimulator \\
Developmental progress & $4 / 14$ \\
& $4 / 14$ virtually none \\
Unable to sit unaided & Some experienced a regression or multiple regressions \\
Able to walk (ever) & $7 / 14$ \\
No speech & $4 / 14$ \\
Microcephaly & $11 / 14$ (very limited in 2) \\
Severe intellectual disability & $8 / 14$ \\
Hand stereotypies & $13 / 14$ \\
Autonomic features & $9 / 14$ \\
\hline
\end{tabular}

Two brothers with a deletion encompassing RS1 and the last exon of CDKL5 were not included as limited clinical information was available. ${ }^{13}$ Additional clinical details kindly provided by Dr Van Esch - personal communication.

The phenotype associated with a CDKL5 mutation ranges from ISSX and infantile epileptic encephalopathy to atypical RTT plus one case of autism. At the most severe end of the spectrum, two female patients have been described who did not express any normal CDKL5 because one copy of the gene was disrupted by a translocation and the normal copy was inactive due to completely skewed Xinactivation. ${ }^{1}$ These girls were very severely affected and died aged 3 and 16 years. Similarly, a male with a c.183delT mutation was very severely affected and died aged 16 years. ${ }^{2}$ Interestingly, one of his identical twin sisters with the same CDKL5 mutation was the most mildly affected patient reported with a diagnosis of autism. The other twin was moderately affected and had a diagnosis of atypical RTT. The difference in phenotype between the twins did not appear to be due to X-inactivation status. Patients with missense mutations were relatively mildly affected, for example they learned to walk (Tao et $\mathrm{al}^{3}$ and patient 3 , this study). The phenotype associated with truncating mutations was variable and did not clearly correlate with the position of the mutation. Clearer genotype-phenotype correlations may become apparent as more mutations are found.

It is interesting that the two patients who had an implanted vagus nerve stimulator showed a reduction in seizure activity, both in terms of frequency and duration of seizures. This may suggest that there is an autonomic component to their seizure disorder. In addition, there were other reported features that could have an autonomic origin including small, cold feet (four patients) and breathing dysrhythmia (seven patients). Autonomic features are also frequently reported in RTT and it is not uncommon for autonomic seizures to be mistaken for, and treated as, epileptic seizures in these patients. Detailed autonomic studies in patients with CDKL5 mutations would help to determine if there is an autonomic component to their seizure disorder requiring alternative therapeutic strategies and whether an implanted vagusnerve stimulator may be of benefit to further patients with CDKL5 mutations.

No CDKL5 mutations have so far been found in classic RTT cases. Also, CDKL5 mutations appear to be less common in males than in female subjects (although fewer males have been tested so far; 16 in this study and one in Weaving et $a l^{2}$ ). It remains to be determined whether CDKL5 mutations are normally lethal in males. Mutations in $C D K L 5$ are relatively common in patients whose seizures begin before the age of 6 months (3/17 in this study). This is much more frequent than $A R X$ or MECP 2 mutations are in patients with this phenotype. Mutation testing of CDKL5 is indicated in patients with early onset of a severe intractable seizure disorder or idiopathic infantile spasms, and in patients with suspected RTT and early onset seizures. 


\section{Acknowledgements}

We thank the patients, families and clinicians who helped in this study. Thanks also to Julie Maynard and Vikki Humphreys for technical assistance. This work was funded by the Health Foundation, a healthcare charity.

\section{References}

1 Kalscheuer VM, Tao J, Donnelly A et al: Disruption of the serine/ threonine kinase 9 gene causes severe X-linked infantile spasms and mental retardation. Am J Hum Genet 2003; 72: 1401-1411.

2 Weaving LS, Christodoulou J, Williamson SL et al: Mutations of CDKL5 cause a severe neurodevelopmental disorder with infantile spasms and mental retardation. Am J Hum Genet 2004; 75: 1079-1093.

3 Tao J, Van Esch H, Hagedorn-Greiwe M et al: Mutations in the Xlinked cyclin-dependent kinase-like 5 (CDKL5/STK9) gene are associated with severe neurodevelopmental retardation. Am J Hum Genet 2004; 75: 1149-1154.

4 Scala E, Ariani F, Mari F et al: CDKL5/STK9 is mutated in Rett syndrome variant with infantile spasms. J Med Genet 2005; 42: $103-107$.

5 Strømme P, Mangelsdorf ME, Shaw MA et al: Mutations in the human ortholog of Aristaless cause X-linked mental retardation and epilepsy. Nat Genet 2002; 30: 441-445.

6 Weaving LS, Ellaway CJ, Gécz J, Christodoulou J: Rett syndrome: clinical review and genetic update. J Med Genet 2005; 42: 1-7.
7 Kammoun F, de Roux N, Boespflug-Tanguy O et al: Screening of $M E C P 2$ coding sequence in patients with phenotypes of decreasing likelihood for Rett syndrome: a cohort of 171 cases. J Med Genet 2004; 41: e85.

8 Hanefeld F: The clinical pattern of the Rett syndrome. Brain Dev 1985; 7: 320-325.

9 Goutieres F, Aicardi J: Atypical forms of Rett syndrome. Am J Med Genet 1985; S1: $93-94$

10 Evans JC, Archer HL, Whatley SD, Kerr A, Clarke A, Butler R: Variation in exon 1 coding region and promoter of MECP2 in Rett syndrome and controls. Eur J Hum Genet 2005; 13: 124-126.

11 Allen RC, Zoghbi HY, Moseley AB, Rosenblatt HM, Belmont JW: Methylation of HpaII and Hha sites near the polymorphic CAG repeat in the human androgen-receptor gene correlates with X chromosome inactivation. Am J Hum Genet 1992; 51: $1229-1239$.

12 Hagberg B, Hanefeld F, Percy A, Skjedal O: An update on clinically applicable diagnostic criteria in Rett syndrome. Eur I Paediatr Neurol 2002; 6: 293-297.

13 Huopaniemi L, Tyynismaa H, Rantala A, Rosenberg T, Alitalo T: Characterization of two unusual RS1 gene deletions segregating in Danish retinoschisis families. Hum Mutat 2000; 16: 307-314.

14 Villard L, Kpebe A, Cardoso C, Chelly B, Tardieu P, Fontes M: Two affected boys in a Rett syndrome family. Neurol 2000; 55: $1188-1193$

15 Steffenburg U, Hagberg G, Hagberg B: Epilepsy in a representative series of Rett Syndrome. Acta Paediatr 2001; 90: 34-39.

Supplementary Information accompanies the paper on European Journal of Human Genetics website (http://www.nature.com/ejhg) 